\title{
Sex-related differences in plantarflexor function during repeated stretch-shortening cycle loading
}

\author{
Laura-Anne M Furlong ${ }^{1}$ \\ Andrew J Harrison ${ }^{2}$ \\ ${ }^{1}$ National Centre for Sport and Exercise Medicine, \\ Loughborough, Leicestershire, UK; \\ School of Sport, Exercise and Health Sciences, \\ Loughborough University, Leicestershire, UK \\ 2 Physical Education and Sports Sciences Depart- \\ ment, University of Limerick, Limerick, Ireland
}

Corresponding author:

Laura-Anne M Furlong

National Centre for Sport and Exercise Medicine, School of Sport, Exercise and Health Sciences,

Loughborough University

LE113 TU, Leicestershire, UK

Tel.: +44 (0) 1509223059

E-mail: L.A.M.Furlong@lboro.ac.uk

\section{Summary}

Introduction: Differences between male and female muscle-tendon units have been previously observed. It is unknown if a sex-related difference exists in the plantarflexor response to repeated stretch-shortening cycles, as occurs during activities of daily living such as walking and running. Methods: An adapted force sledge was used with three dimensional motion analysis to investigate the response of the plantarflexors of 34 age and training-matched males and females during stretchshortening cycle impacts.

Results: Contact times and flight times were found to be similar between groups. Statistically significant differences in absolute peak force and rates of force development were observed during loading. With normalisation to plantarflexor muscle volume, small and moderate effect sizes were observed for all force and rate of force development variables but only peak force remained statistically significant. Differences in absolute stiffness, peak negative and positive power and work were statistically significant with moderate effect size, but with normalisation only differences in peak negative power and work remained statistically significant with a moderate effect size.

Conclusion: These results show females have low- er force, rate of force production and force absorption capabilities, which are of relevance in injury prevention and rehabilitation, and informing personalised engineering design.

Level of evidence: III b (individual case-control study).

KEY WORDS: anterior cruciate ligament, assistive technology, eccentric, gender, jumping, locomotion, running.

\section{Introduction}

Understanding fundamental in vivo mechanical behaviour of muscle is an essential component of development and optimisation of injury prevention, rehabilitation, and training programmes, as well as design of assistive technology such as prosthetics or exoskeletons. The plantarflexor muscle group has a vital role to play in gait, for everyday tasks of daily living such as walking and running, and for maximal performance in many sports. It is important for maintaining postural control, is responsible for the push-off phase of gait and acts to both produce power and stabilise the limb during acceleration and maximal velocity running. When task demands increase, (e.g. with decreased ground contact time) the ankle joint and its surrounding plantarflexor musculature play an increasingly important role in maintaining the overall mechanical stiffness and performance output of the entire limb 1,2. Bipedal gait consists of multiple stretch-shortening cycles (SSC) within the muscle-tendon unit (MTU), whereby an eccentric contraction immediately precedes a concentric contraction, improving the force generation of the concentric contraction. SSC function is affected by underlying MTU mechanics and modifications in the muscle activation strategy used, both of which may vary due to training ${ }^{3}$ or an individual's sex ${ }^{4,5}$. Mechanical stiffness of the MTU and its constituent structures may influence SSC performance, and while some differences may be due to body mass, previous work has shown a decreased ability of females to effectively utilise the SSC in the upper body even when normalised to body mass ${ }^{6}$. These results suggest male and female MTUs behave differently, with only some differences attributed to size differences. Therefore, differences must also be present in the underpinning mechanical behaviour during this type of loading. Previous work has shown females rely more on the plantarflexors than the hip muscles for 
impact absorption during single leg landing ${ }^{7}$ which potentially places this muscle group and the two joints it crosses (i.e. the ankle and knee) at increased risk of injury. Improving understanding of how in vivo mechanical behaviour of muscle groups varies with sex is essential to gain further insight into both injury and performance related differences that have been previously observed, and to establish if this is potentially an important characteristic which needs to be addressed in aspects of personalised engineering design.

Recent work has developed an adapted force sledgebased method of isolating the plantarflexors in analysis of a SSC task. The method has been shown to provide good movement control and reliability, while maintaining validity as a dynamic SSC task ${ }^{8}$. Use of this adapted sledge could provide important information on differences in the plantarflexor responses to cyclical SSC loading due to sex. The aim of this study was to identify differences in plantarflexor peak force, rates of force development, stiffness, power and work in males and females during a cyclical loading task performed on an adapted sledge system. This can provide useful insight into how the male and female MTU differs by understanding their force and energy absorption and generation capacities, which is of benefit for those working in injury prevention, rehabilitation, elite sporting performance, and engineering of assistive technology.

\section{Materials and methods}

\section{Participants}

Following university research ethics approval, 17 males (age: $23.2 \pm 2.73$ years; height: $1.78 \pm 0.05 \mathrm{~m}$; mass: $80.8 \pm 7.81 \mathrm{~kg}$ ) and 17 females (age: $24.2 \pm 1.87$ years; height: $1.68 \pm 0.07 \mathrm{~m}$; mass: $65.4 \pm 6.90 \mathrm{~kg}$ ) gave written, informed consent to participate in this study $^{9}$. Male and female groups were matched for age within 18 months and training background. Each group contained seven field sports athletes, eight recreational athletes who participated in physical activity for at least 30 minutes a day, 4 days per week and two sprint-trained athletes who trained using similar plyometric exercises one day per week. No participant had a history of lower limb surgery or was taking any medications. All participants were free from lower limb injury for the preceding 3 months and were advised not to participate in any unaccustomed strenuous exercise for the 24 hours preceding data collection.

\section{Test protocol}

An adapted force sledge apparatus consisting of a rigid wooden plate, free to move along rails angled at $30^{\circ}$ to the horizontal was used to determine plantarflexor function during the SSC task ${ }^{8}$. A $9.5 \mathrm{~mm}$ retroreflective marker placed on the side of this plate was tracked by three, dimensional infra-red motion analysis cameras $(50 \mathrm{~Hz}$, MAC Eagle, Motion Analysis Corporation Inc., Santa Rosa, CA., USA). The ac- celeration of this marker was used for subsequent calculation of resultant force acting on the foot. Participants were positioned supine at the base of the sledge with the thigh secured to a solid support at its proximal and distal ends. The ankle joint of the preferred hopping limb was free to move and pushed the plate up the inclined rails while the knee angle remained relatively constant throughout the movement, (at approximately $150^{\circ}$ to $170^{\circ}$ ) during impact. Knee movement was constrained as much as practically possible, to ensure the majority of muscle force generation and absorption was isolated to the plantarflexor muscle group.

Participants were instructed to strike the plate rhythmically within a marked area pushing it as hard and as fast as possible, using only the ankle joint. Participants were initially asked to push the plate up the sledge rails using the above criteria and then the plate was dropped towards their foot from a $30 \mathrm{~cm}$ height after a "3, 2, 1" countdown. This protocol was repeated for a minimum of 25-30 impacts until the participant was confident in performing the task correctly and the researcher judged them to be striking the plate as instructed. This contact was always initially with the forefoot due to the orientation of the sledge rails to the ground.

The ultimate aim of initial trials was to achieve the maximum loading a participant could exert for $11 \mathrm{im}$ pacts. Participants were allowed two minutes rest between each series of impacts but if more time was required this was allowed. Similar to the practice trials, the plate began at a position $30 \mathrm{~cm}$ above the foot and was released after a " $3,2,1$ " countdown. Participants were instructed to continue striking the plate for a total of 11 impacts. Following each series of $11 \mathrm{im}$ pacts, the plate was secured away from the foot and additional mass added to the sledge. A loading equivalent to $70 \%$ of this 11 repetition maximum (RM) was used for the test trial as this has previously been shown to be reliable in measurement of peak force $\left(F_{P}\right)$, contact time $(C T)$ and flight time $(F T)$, and a loading that participants were comfortable with using $^{8}$. This maximum loading was typically achieved within 5-6 trials. All trials (familiarisation, 11 repetition maximum, final test effort) were completed in one test session.

\section{Data treatment and variable definition}

The coordinates of the sledge marker were filtered using a fourth order, zero lag, low-pass Butterworth filter. Residual analysis was conducted to identify the optimum cut-off frequency to ensure an optimal signal to noise ratio, which occurred at a cut-off frequency of $12 \mathrm{~Hz}$. A visual check was also performed to ensure the impact was not excessively smoothed by use of this cut-off frequency. Filtering was completed in Cortex software (v3.1.1.1290, Motion Analysis Corporation Inc., Santa Rosa, CA., USA). All variables were calculated in Microsoft Excel (Microsoft Inc., Redmond, WA., USA).

The $5^{\text {th }}$ to $7^{\text {th }}$ impacts were considered representative 
of the plantarflexor response to cyclical SSC loading, and have previously been shown to be consistent and reliable for analysis of the variables of interest ${ }^{8}$. The sledge plate acceleration was calculated as the second derivative of plate position with respect to time, with force calculated using Newton's second law using a correction for the component of weight acting down the sledge rails as the sledge was angled at $30^{\circ}$. CT was defined as the period when plate marker acceleration was greater than zero and FT defined as the period when it was zero or less (Fig. 1). These definitions were used assuming that when the sledge was not in contact with the foot it was in free-fall, therefore the only forces acting on it were the weight component due to gravity $\left(-4.905 \mathrm{~m} . \mathrm{s}^{2}\right)$ and friction. The rolling friction of the sledge on the rails during free-fall was calculated from previous experiments as $0.18 \%$ so was considered negligible for these calculations. This value indicates the calculated acceleration of the sledge plate in contact with the rails was $0.18 \%$ different to that of the plate in freefall. $F_{P}$ was the maximum force developed during each contact with the timing of $F_{P}(\% C T)$ referring to the percentage of CT where $F_{P}$ occurred. Rate of peak force development (RPFD) was calculated as the $F_{P}$ divided by the time in seconds it took to reach it. Rate of force development in the first 30 and $50 \mathrm{~ms}\left(\mathrm{RFD}_{0-30}, \mathrm{RFD}_{0-50}\right)$ was calculated as the force at 30 and $50 \mathrm{~ms}$ post-initial contact, divided by 0.03 and 0.05 s respectively.

The stiffness of the plantarflexor MTU $\left(K_{P F}\right)$ was calculated as $F_{P}$ divided by the peak displacement of the sledge in metres during contact in the direction of the sledge rails. Power was calculated as the product of sledge force and sledge velocity (measurement unit: watts, W), with work done calculated as the time integral of power (measurement unit: joules, J). The negative phase was defined as the period when power was negative, and the positive phase defined as the period when power was positive. Peak power $\left(\mathrm{P}_{\mathrm{NEG}}\right.$, P POS), timing of peak power and total work done ( $\left.\mathrm{W}_{\mathrm{NEG}}, \mathrm{W}_{\mathrm{POS}}\right)$ were calculated for each phase, with time expressed as a percentage of the entire CT. The plate height (i.e. displacement from release to peak of flight) was calculated using the equations of uniformly accelerated motion, assuming equal periods of upward and downward flight.

The influence of increased muscle size on force production has been previously established, therefore all force-related variables were normalised to total plantarflexor muscle volume by dividing observed force

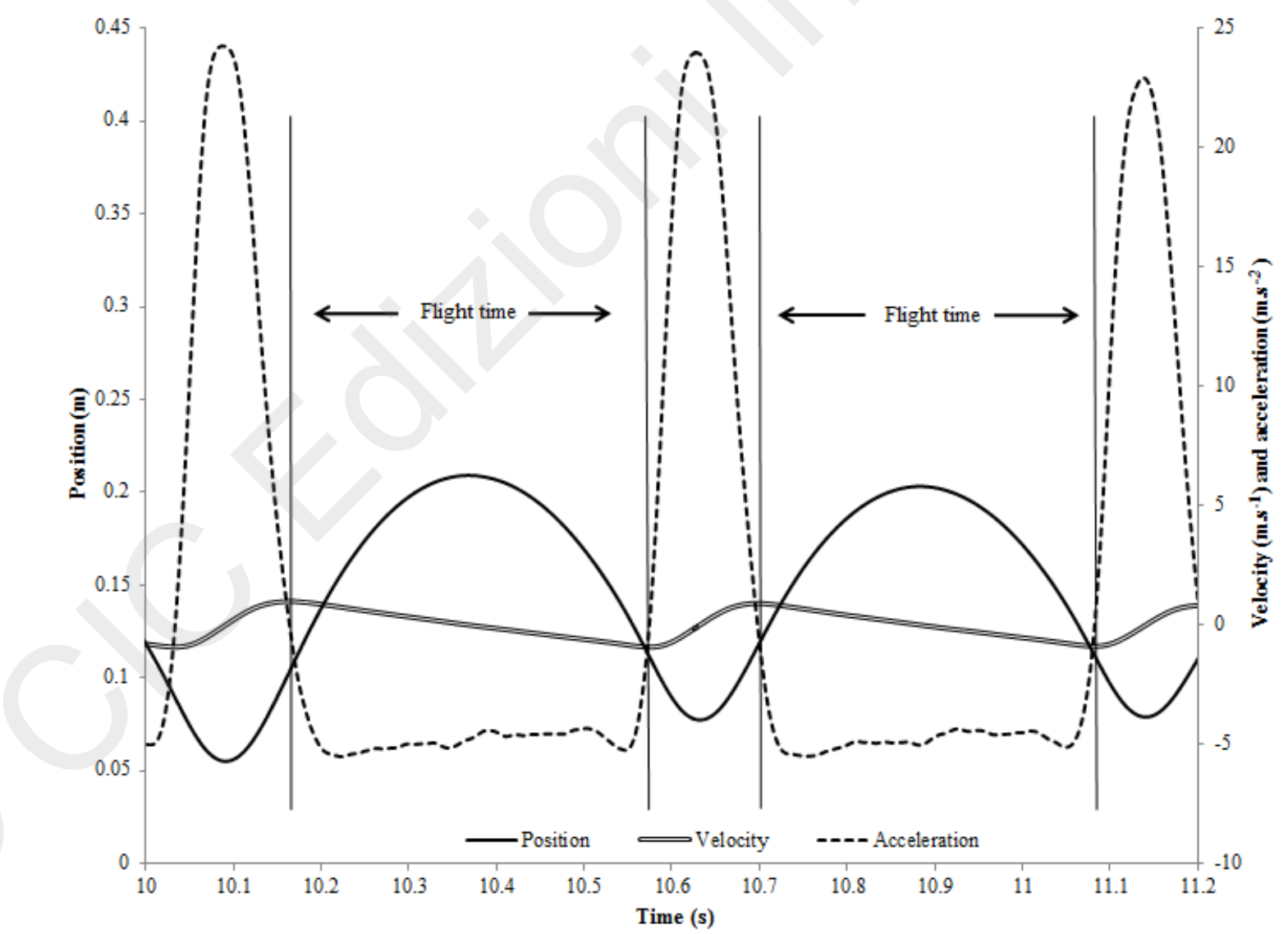

Figure 1. CT and FT were determined using sledge marker kinematics. CT was defined as the time period when sledge acceleration was greater than 0 m.s. ${ }^{2}$ and FT as the time between consecutive CT. These instances occur simultaneously with times when sledge position was at a minimum. As force was calculated as the product of sledge mass and acceleration, peak force was the instant of peak acceleration during the contact. 
measures by personalised total plantarflexor muscle volume. This volume was calculated using the regression equations of Handsfield et al. ${ }^{10}$ for soleus, gastrocnemius medialis and lateralis, peroneus longus and brevis, tibialis posterior, flexor hallucis longus and flexor digitorum longus and based on height and mass products. Volume was considered a suitable variable for normalisation due to its relationship to muscle physiological cross-sectional area (PCSA) which has an established relationship with force-producing capacity ${ }^{11}$. While normalisation to physiological cross-sectional area is acknowledged as the most appropriate method to scale muscle forces, it is very difficult to accurately measure individualised muscle volume, muscle fibre length and muscle pennation angle without additional expensive, highly technical equipment and methods. Muscle volume has been used previously in the literature to account for differences in size between individuals particularly in tasks using isolated muscle groups. Both absolute and normalised values are presented in the results.

\section{Statistical analysis}

The mean absolute and normalised values for each variable from the three impacts for each participant were used for statistical analyses. The means and standard deviations for each sex group were calculated for each variable to provide normative values and estimate of sample variability. Groups were considered well-matched since similar coefficients of variation were observed in each group. The reliability of these measures was determined by calculating the average within-participant standard deviation and coefficient of variation across the three trials and by calculating the average intra-class correlation coefficient (two-way random with absolute agreement). All statistical analyses were completed using SPSS Statistics 20 (IBM, Armonk, NY, USA). Between-group differences were determined using independent samples ttests. The assumptions of this test were checked using Shapiro-Wilk's test of normality and Levene's test of equality of variances. Where data violated the assumptions, differences were determined using a Mann-Whitney $U$ test. Type I error level was set at a $<0.05$. Effect size (practical significance) was calculated using Cohen's d using the formula

$$
\mathrm{d}=\frac{\left|m_{M}-m_{F}\right|}{\sigma^{\prime}}
$$

where $m_{M}$ and $m_{F}$ are the means of the male and female groups respectively, and $\sigma_{\Lambda^{\prime}}$ is the pooled standard deviation of the two groups ${ }^{12}$. The scale for classification of effect size was $<0.2=$ trivial, 0.2 to $0.6=$ small, 0.6 to $1.2=$ moderate, 1.2 and above $=$ large $^{13}$. Moderate and large effects were considered practically significant.

\section{Results}

\section{Measurement reliability}

Variables were shown to be highly reliable for both males and females, with all average ICCs greater than .806 and most ICCs greater than .900 . Withinparticipant variability was also low, with almost all coefficients of variation below $12 \%$. The highest variability was observed in RPFD and RFD $0-30$ (Tab. I).

\section{Contact time and timing of peak events}

Parametric statistical tests were used to determine between-group differences for $F_{T}$ and normalised $F_{P}$, RPFD, RFD 0-50, $W_{N E G}$ and $W_{P O S}$. Non-parametric tests were used for all other variables. During cyclical loading, no practical or statistically significant between-group differences were observed in how the participants struck the plate (as defined by CT or \% CT when $F_{P}$ occurred). CT was indicative of a fast SSC, with CT of $0.183 \pm 0.038 s$ and $0.174 \pm 0.035 s$ (mean \pm standard deviation) observed in males and females respectively. This difference was less than $5 \%$ and neither practically nor statistically significant. The timing of $\mathrm{F}_{\mathrm{P}}$ (males: $41.7 \pm 4.5 \% \mathrm{CT}$; females: $40.8 \pm 4.8 \% \mathrm{CT}$ ), $\mathrm{P}_{\mathrm{NEG}}$ (males: $23.1 \pm 2.8 \% \mathrm{CT}$, females: $22.7 \pm 2.3 \% \mathrm{CT}$ ) and PPOS (males: $69.7 \pm 4.2 \%$ CT; females: $72.3 \pm 4.5 \% \mathrm{CT}$ ) were similar for males and females with non-significant differences of negligible and small effect size observed.

\section{Muscle volume, peak force, and rate of force development}

Statistically significant differences in calculated total plantarflexor muscle volume of large effect size were observed $\left(1086 \pm 113 \mathrm{~cm}^{3}\right.$ for females, $1360 \pm 122 \mathrm{~cm} 3$ for males). Statistically significant between-group differences with moderate (tending towards large) or large effect sizes were observed in all absolute measures of force and force development ( $F_{P}, R P F D$, $\left.\mathrm{RFD}_{0-30}, \mathrm{RFD}_{0-50}\right)$. With normalisation, the differences were small to moderate in size but only the difference in $F_{P}$ remained statistically significant (Tab. II).

\section{Stiffness, power, and work}

Statistically significant differences of moderate effect size were observed in absolute measures of stiffness, peak power, and work in both negative and positive phases (Tab. III). With normalisation to muscle volume, differences in $K_{P F}$ became negligible. Similarly, $P_{\text {POS }}$ and $W_{\text {Pos }}$ were no longer statistically significant and of small effect size. $P_{\text {NEG }}$ and $W_{N E G}$ remained statistically significant and of moderate effect size.

\section{Discussion}

The results of this study showed moderate to large differences in the plantarflexor force, force production and mechanical behaviour of males and females during repeated SSC loading in a sample of healthy young adults matched for age and training background. This was despite no significant differences in CT or timing of $F_{P}, P_{N E G}$ and P POs. This shows comparable strategies were used by both sexes to absorb and generate force. While some differences were re- 


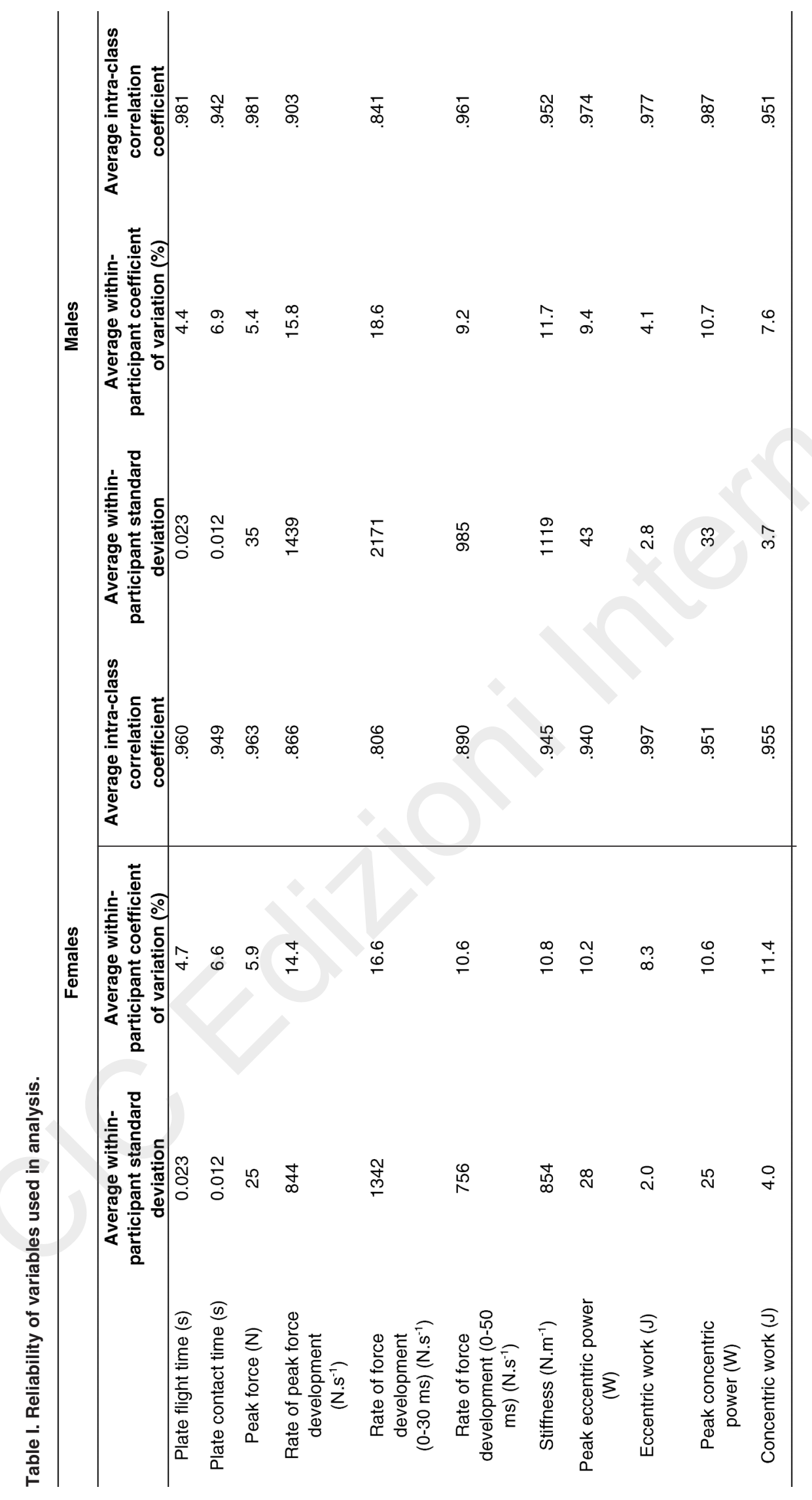



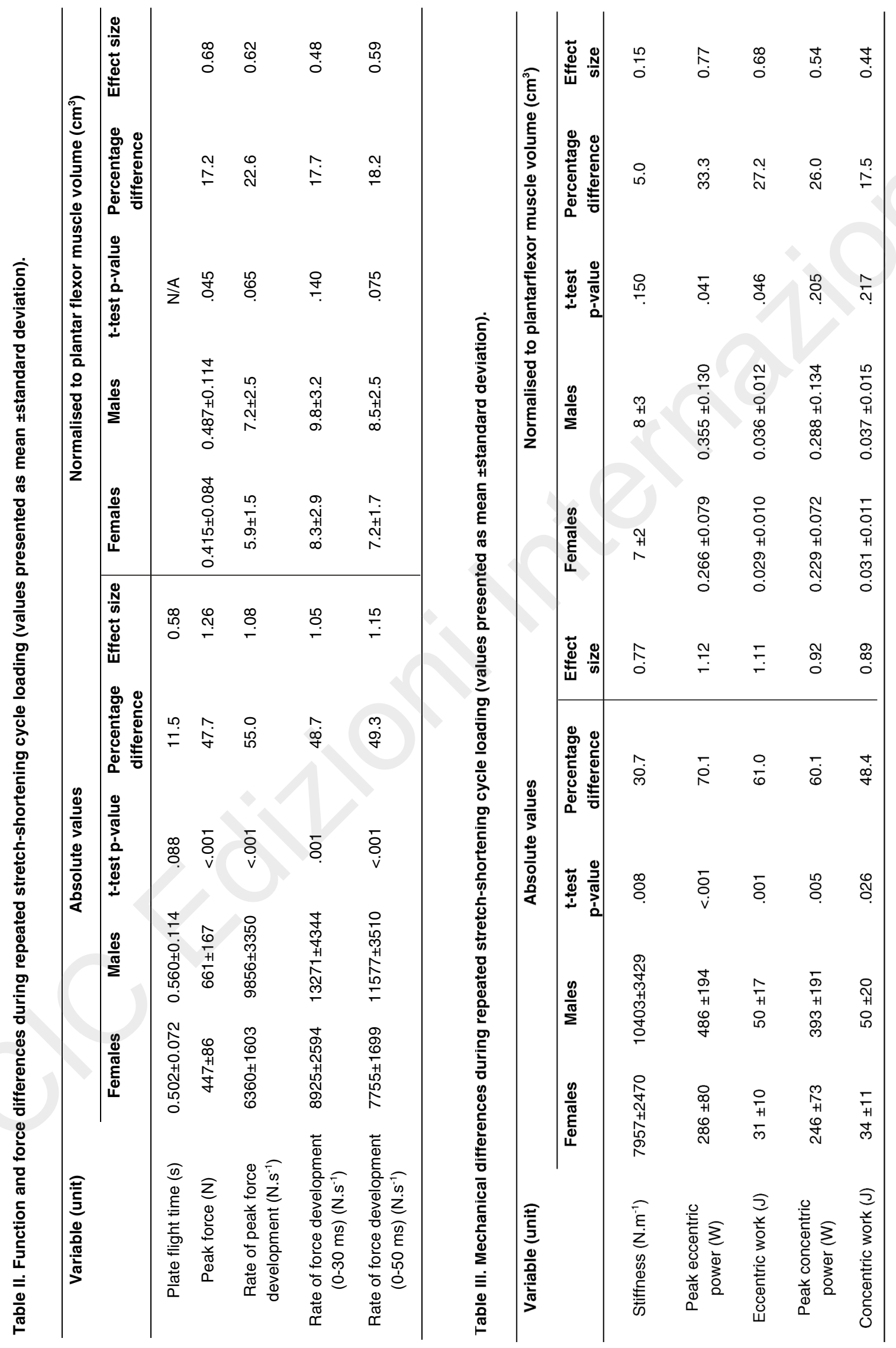
moved with normalisation to muscle volume, several other important measures remained statistically and practically significant.

The lower absolute $F_{P}, R P F D, R F D_{0-30}$ and $R F D_{0-50}$ observed in females is similar to previous work but this study is one of the first to establish this in a dynamic, cyclical SSC task. With normalisation, these differences became smaller but were still of moderate effect size. Males have been shown to have greater muscle thickness (known to be related to increased muscle size), and increased pennation angle than females in both soleus and gastrocnemius ${ }^{14}$, both of which increase force production capacity and may account for differences observed. Muscle volume differences were statistically and practically significant with a large effect size and this may partly explain observed differences, however, not all differences were removed with normalisation, therefore other factors must also have affected these measures. Males are known to be more powerful due to increased testosterone and/or an improved ability to use the SSC effectively ${ }^{6}$. The SSC is thought to be more effective with increased tendon stiffness and males have been shown to have a stiffer Achilles tendon than females of similar training background, probably due to a lack of circulating oestrogen and increased muscle mass. Increased tendon stiffness shifts the force-velocity curve to the right so for the same muscle contraction velocity, force production capabilities are higher. This could explain, at least in part, why higher forces and rates of force development were observed in male participants.

Absolute rates of force development during both tasks were significantly lower in females. With normalisation, these differences were still of moderate effect size. Differences in RPFD and RFD $0-50$ were of greater effect size than $R_{F} D_{0-30}$, possibly due to increased contribution of muscle activation in these variables and the decreased ability of females to develop force rapidly. These results are of particular interest in relation to the role of the plantarflexors in prevention of serious knee injury since injury occurs when the magnitude of loading exceeds the level of loading the tissue of interest can withstand. While much literature focuses on the role of the hamstring and quadriceps muscle groups in anterior cruciate ligament $(A C L)$ injury ${ }^{15}$, several Authors have suggested the plantarflexors are also important ${ }^{16-18}$. While knee valgus-varus moments are often considered the main cause of ACL injury, Yu and Garrett ${ }^{19}$ presented strong evidence for the role of sagittal plane kinematics and anterior tibial translation as the primary cause of non-contact ACL injury. Knee valgus-varus moments appear most important when occurring in conjunction with high levels of anterior tibial translation. Gastrocnemius has been shown to be an antagonist of the ACL, acting to increase anterior tibial translation $^{16}$ but soleus has been shown to exert posterior forces on the tibia due to its anatomical origin ${ }^{17}$. As the physiological cross-sectional area of soleus is approximately 3-8 times larger than the gastrocne- mius $^{20}$, this suggests the plantarflexors as a group will act to reduce ACL loading. As ACL injuries are most likely to occur 17 to $50 \mathrm{~ms}$ following initial ground contact ${ }^{21}$, the deficit in rates of force development of females observed during this time period in this study may provide a further explanation why females are more likely to damage their ACL. While this may be due in part to muscle activation differences, the normalised results clearly indicate underlying rate of force development characteristics in surrounding musculature as a further consideration.

A statistically significant difference in absolute $\mathrm{K}_{\mathrm{PF}}$ was observed, which was not present with normalisation. This is similar to findings in other studies ${ }^{22,23}$, and highlights the importance of muscle size and volume in the modulation of stiffness. No difference was observed in normalised Ppos or Wpos, which contrasts to previous work which found increased concentric (positive) power in males during a countermovement jump even after normalisation to body mass 24, and which is due to the movement velocities used. During the negative phase, where muscles are typically working eccentrically, muscle activation has less influence on subsequent power which explains the lack of difference during that phase. The results of this study showed negative power and work to be lower in females even after normalisation, due in part to the smaller forces generated. These differences may also be due to previously reported differences in tendon stiffness, fibre size and/or pennation angle in females. Decreased $P_{\text {NEG }}$ indicates a decreased ability to respond in situations requiring high forces and rapid execution such as landing ${ }^{24}$ and decreased ability to dissipate force, which is concerning as females have previously been shown to preferentially rely on the plantarflexors for impact absorption during landing ${ }^{7}$. This may also predispose females to injury during impact-related tasks. This result also highlights a novel consideration in the design of assistive technologies such as prosthetics or exoskeletons. While females have been shown to preferentially utilise the plantarflexors for impact absorption during landing, less is known about this during gait. The females in this study demonstrated a decreased ability to absorb force during cyclical SSC loading, as which may occur during walking and running. Future work to incorporate these findings into studies examining force dissipation during gait would guide engineering design into the relative importance of this.

Males tend to have larger body and muscle mass than females, therefore scaling of data to account for differences in body size is necessary to determine if meaningful differences truly exist. Normalisation to body mass or a power of body mass is commonly used for scaling of forces in tasks such as jumping or running as the entire body has an influence on the force output. Caution has been advised on using body mass as the normalisation variable in isolated muscle tasks as there is no definitive relationship between increased body mass and increased specific muscle mass ${ }^{25}$. As a result, force for isolated muscle 
groups is often normalised to physiological cross-sectional area or muscle volume. Muscle volume is most accurately determined by magnetic resonance imaging or computed tomography, but this facility is not generally available for non-clinical use. The regression equations used in this study are based on height and mass products and account for between 21 and $66 \%$ of individual plantarflexor muscle volumes. While there is still a large proportion of unaccounted variance, this calculated muscle volume appears to be more suitable for normalisation rather than solely body mass. The findings of Handsfield et al. support the inconsistent relationship between body mass and individual muscle volume as they found equations based solely on body mass performed worse in the prediction of muscle volume. There are some limitations to its use in this study, such as its generic, nonsex-specific nature. Due to the typical differences in body composition for males and females, even with similar activity levels, it is possible some errors may be present and therefore sex-specific regression analyses may be more suitable for prediction of muscle volumes. It is debatable whether allometric scaling or a ratio method should be used for normalisation; there were several highly active individuals in this study, so their predicted muscle volumes may be prone to errors. Use of a linear normalisation of force to muscle volume was the most appropriate analysis for this data set as the definitive answer to these problems remains unclear.

The results of this study show males have a greater force production and rate of force production capability than females during cyclical SSC loading. Large differences were also present in the mechanical properties of the plantarflexors in stiffness, work, and power. While some of these variables were not significant when normalised, these results may explain, at least in part, why there is an increased risk of injury in females, suggest potential areas of focus in injury prevention or rehabilitation strategies, and highlight areas for potential consideration by the engineering professional.

\section{Acknowledgement}

This research was funded by the Irish Research Council under the Embark Initiative.

\section{References}

1. Hobara H, Kanosue K, Suzuki S. Changes in muscle activity with increase in leg stiffness during hopping. Neurosci Lett. 2007;418:55-59.

2. Hobara H, Inoue K, Omuro K, Muraoka T, Kanosue K. Determinant of leg stiffness during hopping is frequency-dependent. European Journal of Applied Physiology. 2011;111:21952201.

3. Padua D, Carcia C, Arnold B, Granata K. Gender differences in leg stiffness and stiffness recruitment strategy during two- legged hopping. Journal of Motor Behavior. 2005;37(2):111126.

4. Blackburn JT, Padua DA, Weinhold PS, Guskiewicz KM. Comparison of triceps surae structural stiffness and material modulus across sex. Clin Biomech. 2006;21(2):159-167.

5. Hoge KM, Ryan ED, Costa PB, Herda TJ, Walter AA, Stout JR, et al. Gender differences in musculotendinous stiffness and range of motion after an acute bout of stretching. J Strength Cond Res. 2010;24(10):2618-2626 .

6. Miyaguchi K, Demura S. Gender difference in ability using the stretch-shortening cycle in the upper extremities. J Strength Cond Res. 2009;23(1):231-236.

7. Weinhandl J, Joshi M, O'Connor KM. Gender comparisons between unilateral and bilateral landings. J Appl Biomech. 2010;26:444-453.

8. Furlong L, Harrison A. Reliability and consistency of plantarflexor stretch-shortening cycle function using an adapted force sledge apparatus. Physiol Meas. 2013;34(4):437-448.

9. Padulo J, Oliva F, Frizziero A, Maffulli N. Muscles, Ligaments and Tendons Journal - Basic principles and recommendations in clinical and field science research: 2016 update. MLTJ. 2016;6(1):1-5.

10. Handsfield G, Meyer C, Hart J, Abel M, Blemker S. Relationships of 35 lower limb muscles to height and body mass quantified using MRI. J Biomech. 2014;47(3):631-638.

11. Powell $P$, Roy R, Kanim P, Bello M, Edgerton V. Predictability of skeletal muscle tension from architectural determinations in guinea pig hindlimbs. J Appl Physiol. 1984;57:1715-1721.

12. Cohen J. Statistical Power Analysis for the Behavioral Sciences. Revised ed. New York: Academic Press Inc.; 1977.

13. Hopkins W. A New View of Statistics: A Scale of Magnitudes for Effect Sizes. Available from: http://www.sportsci.org/resource/stats/effectmag.html.

14. Chow R, Medri M, Martin D, Leekam R, Agur A, McKee N. Sonographic studies of human soleus and gastrocnemius architecture: gender variability. Eur J Appl Physiol. 2000;82:236244.

15. Oberhofer K, Hosseini Nabab S, Schutz P, Postolka B, Snedeker J, Taylor W, et al. The influence of muscle-tendon forces on ACL loading during jump landing: a systematic review. Muscles, Ligaments and Tendons Journal. 2017;7(1): 125-135.

16. Fleming B, Renstrom P, Ohlen G, Johnson R, Peura G, Beynnon $B$, et al. The gastrocnemius muscle is an antagonist of the anterior cruciate ligament. J Orthop Res. 2001;19:1178-1184.

17. Elias J, Faust A, Chu Y, Chao E, Cosgarea A. The soleus muscle acts as an agonist for the anterior cruciate ligament. Am J Sports Med. 2003;31:241-246.

18. Mokhtarzadeh H, Yeow C, Goh J, Oetomo D, Malekipour F, Lee P. Contributions of the soleus and gastrocnemius muscles to the anterior cruciate ligament loading during single-leg landing. J Biomech. 2013;46:1913-1920.

19. Yu B, Garrett W. Mechanisms of non-contact $A C L$ injuries. $B r$ J Sports Med. 2007;41(S1):147-151.

20. Fukunaga T, Roy R, Shellock F, Hodgson J, Day M, Lee P, et al. Physiological cross-sectional area of human leg muscles based on magnetic resonance imaging. J Orthop Res. 1992;10(6):926-934.

21. Krosshaug T, Nakamae A, Boden B, Engebretsen L, Smith G, Slauterbeck J, et al. Mechanisms of anterior cruciate ligament injury in basketball: video analysis of 39 cases. Am J Sports Med. 2007;35(3):359-367.

22. Granata KP, Padua DA, Wilson SE. Gender differences in active musculoskeletal stiffness. Part II. Quantification of leg stiffness during functional hopping tasks. J Electromyogr Kinesiol. 2002;12(2):127-135. 
23. Padua DA, Arnold BL, Perrin DH, Gansneder BM, Carcia CR, Granata KP. Fatigue, vertical leg stiffness, and stiffness control strategies in males and females. J Athl Train. 2006;41(3):294304.

24. Caserotti P, Aagard P, Simonsen E, Puggaard L. Contractionspecific differences in maximal muscle power during stretch- shortening cycle movements in elderly males and females. Eur J Appl Physiol. 2001;84:206-212.

25. Nevill A, Stewart A, Olds T, Holder R. Are adult physiques geometrically similar? The dangers of allometric scaling using body mass power laws. Am J Phys Anthropol. 2004;124:177182. 